\title{
Ammonia Mediates Cortical Hemichannel Dysfunction in Rodent Models of Chronic Liver Disease
}

Anna Hadjihambi, ${ }^{1,2}$ Francesco De Chiara, ${ }^{1}$ Patrick S. Hosford, ${ }^{2}$ Abeba Habtetion, ${ }^{1}$

Anastassios Karagiannis, ${ }^{3}$ Nathan Davies, ${ }^{1}$ Alexander V. Gourine, ${ }^{2^{*}}$ and Rajiv Jalan ${ }^{1^{*}}$

The pathogenesis of hepatic encephalopathy (HE) in cirrhosis is multifactorial and ammonia is thought to play a key role. Astroglial dysfunction is known to be present in HE. Astrocytes are extensively connected by gap junctions formed of connexins, which also exist as functional hemichannels allowing exchange of molecules between the cytoplasm and the extracellular milieu. The astrocyte-neuron lactate shuttle hypothesis suggests that neuronal activity is fueled (at least in part) by lactate provided by neighboring astrocytes. We hypothesized that in $\mathrm{HE}$, astroglial dysfunction could impair metabolic communication between astrocytes and neurons. In this study, we determined whether hyperammonemia leads to hemichannel dysfunction and impairs lactate transport in the cerebral cortex using rat models of $\mathrm{HE}$ (bile duct ligation [BDL] and induced hyperammonemia) and also evaluated the effect of ammonia-lowering treatment (ornithine phenylacetate [OP]). Plasma ammonia concentration in BDL rats was significantly reduced by OP treatment. Biosensor recordings demonstrated that $\mathrm{HE}$ is associated with a significant reduction in both tonic and hypoxia-induced lactate release in the cerebral cortex, which was normalized by OP treatment. Cortical dye loading experiments revealed hemichannel dysfunction in HE with improvement following OP treatment, while the expression of key connexins was unaffected. Conclusion: The results of the present study demonstrate that $\mathrm{HE}$ is associated with central nervous system hemichannel dysfunction, with ammonia playing a key role. The data provide evidence of a potential neuronal energy deficit due to impaired hemichannel-mediated lactate transport between astrocytes and neurons as a possible mechanism underlying pathogenesis of HE. (HEPATOLOGY 2017;65:1306-1318)

$\mathrm{H}$ epatic encephalopathy $(\mathrm{HE})$ is a serious neuropsychiatric complication that is associated with liver dysfunction and is diagnosed when other known brain disorders are excluded. ${ }^{(1)} \mathrm{HE}$ comprises a range of symptoms, including sleep disturbances and alterations in cognitive, behavioral, fine motor, and psychomotor functions, with coma and death occurring at the late stages. ${ }^{(2)}$ Several hypotheses regarding the pathogenesis of $\mathrm{HE}$ have been proposed, and numerous factors have been suggested as key players in $\mathrm{HE}$, including inflammation, ${ }^{(3)}$ oxidative stress, ${ }^{(4)}$ impaired brain energy metabolism, ${ }^{(5)}$ and-most commonly - the neurotoxic effects of ammonia. ${ }^{(6)}$

Recent evidence has demonstrated that astroglial lactate production and release in cortical cultures and in the somatosensory cortex of anesthetized rats is

Abbreviations: 4-CIN, $\alpha$-cyano-4-hydroxycinnamic acid; aCSF, artificial cerebrospinal fluid; ALF, acute liver failure; ANOVA, analysis of variance; $B D L$, bile duct ligation; $C B F$, carboxyfluorescein; $C B X$, carbenoxolone; HA, hyperammonemic; HE, hepatic encephalopathy; MCT, monocarboxylate transporter; NPPB, 5-nitro-2-(3-phenylpropylamino) benzoic acid; OP, ornithine phenylacetate.

Received July 27, 2016; accepted December 23, 2016.

Additional Supporting Information may be found at onlinelibrary. wiley.com/doi/10.1002/hep.29031/suppinfo

This study was supported by Grand Challenges UCL and The Wellcome Trust (to Alexander V. Gourine). Alexander V. Gourine is a Wellcome Trust Senior Research Fellow.

*These authors have contributed equally.

Copyright $(2017$ The Authors. HEPATOLOGY published by Wiley Periodicals, Inc., on behalf of the American Association for the Study of Liver Diseases. This is an open access article under the terms of the Creative Commons Attribution License, which permits use, distribution and reproduction in any medium, provided the original work is properly cited.

View this article online at wileyonlinelibrary.com.

DOI 10.1002/hep.29031

Potential conflict of interest: Rajiv Jalan has collaborated with Ocera and Takeda, consults with Ocera, and has received speaking fees from Norgine. He is the inventor of OCR-002, which has been patented by UCL and licensed to Ocera Therapeutics. He is also the founder of Yaqrit Ltd, a spin-off company from the University College London. 
facilitated in the presence of ammonia. ${ }^{(7)}$ This appears to be due to acidification of the mitochondrial matrix resulting in a direct inhibition of mitochondrial pyruvate uptake. Increased brain lactate levels have also been reported in hyperammonemic conditions such as in acute liver failure (ALF), which is thought to be due to inhibition of the tricarboxylic acid cycle enzyme $\alpha$ ketoglutarate dehydrogenase, suggesting a reduction in oxidative metabolism. ${ }^{(8)}$ It remains unknown whether significant changes in brain lactate metabolism develop in conditions of long-term central nervous system exposure to increased ammonia concentrations, such as that seen during chronic liver disease or $\mathrm{HE}$.

Astrocytes, the most numerous glial cells in the central nervous system, are thought to play an important role in HE pathogenesis. The astrocytic dysfunction developing during the progression of the disease could precipitate neuronal pathology, leading to neurological impairment. Astrocytes are extensively connected by gap junctions formed of connexins, which also exist as functional hemichannels allowing effective transfer of ions, metabolic substrates, and signaling molecules across the plasma membrane. ${ }^{(9)}$ Under normal physiological conditions, hemichannels are either closed ${ }^{(10)}$ or in a flickering state. ${ }^{(11)}$ In certain pathological conditions, such as epilepsy and ischemia, significant changes in astroglial structure and function may occur, which are associated with changes in connexin hemichannel function, affecting coupling within the astroglial networks and their communication with other brain cells. ${ }^{(12)}$ We hypothesized, that in $\mathrm{HE}$, connexin hemichannel dysfunction may contribute to the development of its neurological features.

There is recent evidence that hemichannels may function as a conduit of lactate transport across the membrane. ${ }^{(13)}$ In this study, we first investigated whether connexin hemichannel expression and hemichannelmediated release of lactate are altered in animal models of HE. Ornithine phenylacetate (OP, OCR-002; Ocera Therapeutics, CA) has been shown to reduce ammonia levels in animal models of cirrhosis and ALF. OP treatment was found to be associated with a significant reduction in the severity of brain swelling, ${ }^{(14)}$ improvement in neurophysiological function, ${ }^{(15)}$ and reduction in intracranial pressure. ${ }^{(16)}$ Therefore, in this study we applied OP as an experimental ammonia-lowering treatment. The data obtained demonstrate that, in $\mathrm{HE}$, ammonia mediates cortical hemichannel dysfunction associated with a significant reduction in hemichannel-mediated lactate release.

\section{Materials and Methods}

All experiments were performed in accordance with the Animals (Scientific Procedures) Act of 1986, which was revised according to the European Directive 2010/63/EU. All animals received humane care according to the criteria outlined in the Guide for the Care and Use of Laboratory Animals (National Institutes of Health publication 86-23; revised 1985).

\section{ANIMAL MODELS}

Male Sprague-Dawley rats (body weight, 350$400 \mathrm{~g}$ ) were obtained from Charles River Laboratories (Kent, UK).

\section{Bile Duct Ligation Surgery}

Under general anesthesia (5\% isoflurane in 100\% oxygen for induction, $2 \%$ isofluorane in air for

\section{ARTICLE INFORMATION:}

From the ${ }^{1}$ UCL Institute for Liver and Digestive Health, Division of Medicine, UCL Medical School, Royal Free Hospital, Rowland Hill Street, London, United Kingdom; ${ }^{2}$ Centre for Cardiovascular and Metabolic Neuroscience, Neuroscience, Physiology and Pharmacology, University College London, London, United Kingdom; ${ }^{3}$ Neurocentre Magendie, INSERM U1215, Bordeaux, France, University of Bordeaux, Bordeaux, France.

\section{ADDRESS CORRESPONDENCE AND REPRINT REQUESTS TO:}

Rajiv Jalan, M.D., Ph.D.

Liver Failure Group ILDH

Division of Medicine

UCL Medical School, Royal Free Campus

Rowland Hill Street

London, NW3 2PF

United Kingdom

E-mail: r.jalan@ucl.ac.uk

Tel.: + 44-2074332795 or

Alexander V. Gourine, Ph.D.

Neuroscience, Physiology and Pharmacology

Gower Street

London

WC1E 6BT

E-mail: a.gourine@ucl.ac.uk [Correction added March 8, after first online publication: Dr. Gourine's email address was changed from a.gourine@medsch.ucl.ac.uk.] 
maintenance) 30 rats underwent triple ligation of the bile duct (way of a small laparotomy) to induce chronic liver injury and were studied 28 days after surgery. ${ }^{(17)}$

\section{Noncirrhotic Hyperammonemia Condition}

Thirty-two rats were administered a hyperammonemic (HA) diet. The amino acid recipe used for a stock of approximately $100 \mathrm{~g}$ was: $15 \mathrm{~g}$ leucine, $7.7 \mathrm{~g}$ phenylalanine, $7 \mathrm{~g}$ glutamate, $10 \mathrm{~g}$ alanine, $4.4 \mathrm{~g}$ proline, $5.8 \mathrm{~g}$ threonine, $11 \mathrm{~g}$ aspartate, $5 \mathrm{~g}$ serine, $4.8 \mathrm{~g}$ glycine, $3.3 \mathrm{~g}$ arginine, $9.6 \mathrm{~g}$ lysine, $8.4 \mathrm{~g}$ histidine, $3 \mathrm{~g}$ tyrosine, $1.5 \mathrm{~g}$ tryptophan, and $10.6 \mathrm{~g}$ valine. $25 \mathrm{~g}$ of this mix (mixed 1:5 with standard rodent chow powder) was freshly prepared daily with water in a mash form and rats were given free access to it for 5 days. The recipe approximates the amino acid composition of a rodent haemoglobin, ${ }^{(18)}$ mimicking the effect of gastrointestinal bleeding, which is known to result in systemic hyperammonemia. ${ }^{(19)}$

\section{OP Treatment}

Three weeks after surgery, 24 bile duct ligation (BDL)-operated rats were given twice-daily intraperitoneal injections of combined L-ornithine and phenylacetate $(0.3 \mathrm{~g} / \mathrm{kg} ; \mathrm{OP})$ approximately 7 hours apart for 5 days - a regimen that has been shown previously to effectively reduce plasma ammonia concentration. ${ }^{(20)}$ The rats were studied on day 28 after BDL surgery within 3 hours of the last OP injection.

Blood and brain tissue were collected under terminal isoflurane anesthesia. Plasma biochemistry was performed using a Cobas Integra II system (Roche Diagnostics, West Sussex, UK).

\section{Brain Slice Preparation}

Rats were sacrificed humanely by way of isoflurane inhalation overdose. After cardiac perfusion with chilled $\left(4^{\circ} \mathrm{C}\right)$ artificial cerebrospinal fluid $(\mathrm{aCSF}$; $124 \mathrm{mM} \mathrm{NaCl}, 3 \mathrm{mM} \mathrm{KCl}, 2 \mathrm{mM} \mathrm{CaCl}, 26 \mathrm{mM}$ $\mathrm{NaHCO}_{3}, \quad 1.25 \mathrm{mM} \quad \mathrm{NaH}_{2} \mathrm{PO}_{4}, 1 \mathrm{mM} \mathrm{MgSO}_{4}$, $10 \mathrm{mM}$ D-glucose saturated with $95 \% \mathrm{O}_{2}, 5 \% \mathrm{CO}_{2}$, $\mathrm{pH} 7.5, \mathrm{PCO}_{2} 35 \mathrm{mmHg}$ ), with an additional $9 \mathrm{mM}$ $\mathrm{Mg}^{2+}$, the brains were rapidly removed and placed in a bath of chilled $\left(4^{\circ} \mathrm{C}-6^{\circ} \mathrm{C}\right)$ aCSF. Coronal cortical slices $(300 \mu \mathrm{m}$ thick $)$ were cut using a vibrating microtome. The slices were recovered in oxygenated $(95 \%$ $\left.\mathrm{O}_{2}, 5 \% \mathrm{CO}_{2}\right)$ aCSF at room temperature for 30 minutes.

\section{WESTERN BLOT ANALYSIS}

Proteins $(30 \mu \mathrm{g})$ extracted from the cortices of five sham-operated, six BDL, six HA, and five BDL-OP treated rats were separated by way of sodium dodecyl sulphate-polyacrylamide gel electrophoresis on a 4\%12\% Bis-Tris NuPAGE gel (Invitrogen, Scotland, UK) and transferred to nitrocellulose membranes. Membranes were blocked with $5 \%$ bovine serum albumin and incubated with antibodies against connexin43 (Cell Signaling Technology, Danvers, MA; 1:1000), connexin-36 (Santa Cruz Biotechnology, Dallas, TX; 1:1000), connexin-30 (Invitrogen, $1 \mu \mathrm{g} /$ $\mathrm{mL}$ ), and connexin-26 (Thermo Fisher Scientific, Waltham, MA; $1 \mu \mathrm{g} / \mathrm{mL}$ ). Detection of actin (Santa Cruz Biotechnology; 1:1000) was used to control for protein loading. Binding of antibody was detected using a horseradish peroxidase-conjugated secondary antibody (goat anti-rabbit or goat anti-mouse IgGHPR, Santa Cruz Biotechnology; 1:10,000) where appropriate and the SuperSignal Chemiluminescence Substrate for detection of horseradish peroxidase (Pierce, Thermo Fisher Scientific, Waltham, MA). Densitometric analysis was performed using Kodak 1D image analysis software (Kodak, Rochester, NY).

\section{MEASUREMENTS OF LACTATE RELEASE USING MICROELECTRODE BIOSENSORS}

Amperometric enzymatic biosensors were obtained from Sarissa Biomedical (Coventry, UK). The design and operation of the biosensors have been described in detail previously. ${ }^{(21,22)}$ All sensors were operating against a reference electrode $(\mathrm{Ag} / \mathrm{AgCl})$ and had a linear response to lactate within the concentration range recorded in this study. ${ }^{(13,22)}$ Further information on the principle of operation and response time is given in the online data supplement (Supporting Fig. S1A-C). To control for the release of nonspecific electroactive interferants, a dual recording configuration was used. In every recording, a "null" sensor, lacking enzymes but otherwise identical, was used to measure current changes not associated with lactate oxidase activity, which were then subtracted from the current recorded by the lactate biosensor ${ }^{(23)}$ (Fig. 1).

The sensors were calibrated directly in the slice chamber immediately before and after every recording by application of $100 \mu \mathrm{M}$ of lactate (Fig. 1). To convert changes in the biosensor current to changes in lactate concentration, an average of sensor calibrations 


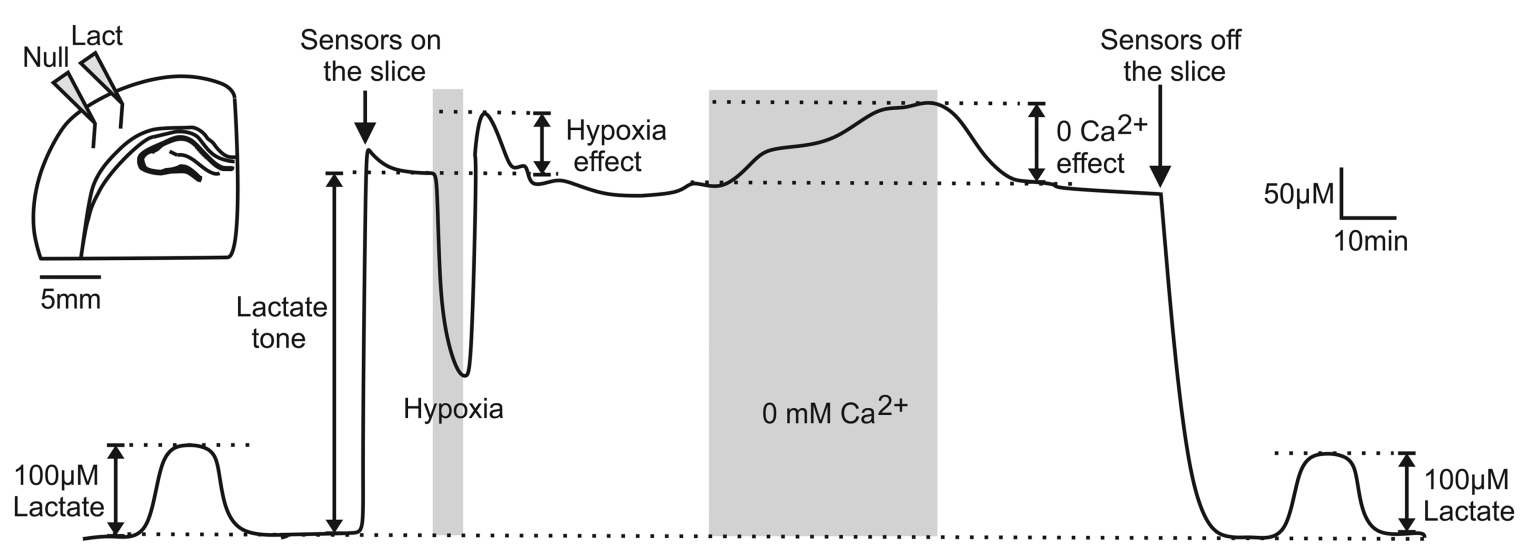

FIG. 1. Measuring release of lactate using microelectrode biosensors. Representative example of changes in the net lactate biosensor current (difference in current between lactate and null sensors) during calibration (100 $\mu \mathrm{M}$ lactate), after biosensor placement in direct contact with the surface of the cortical slice (recording tonic lactate release), in response to a hypoxic challenge (perfusion with aCSF saturated with $95 \% \mathrm{~N}_{2} / 5 \% \mathrm{CO}_{2}$ ), and in response to lowering extracellular $\left[\mathrm{Ca}^{2+}\right]$. Peak hypoxia-induced lactate release is measured upon reoxygenation. Upper left: Schematic drawing of the dual recording configuration of lactate and null (control) biosensors placed on the surface of the brain slice.

before and after the recording were used. For each of the recordings, a slice was transferred into the recording chamber and superfused with $\mathrm{aCSF}$ at $35^{\circ} \mathrm{C}$ $\left(3 \mathrm{~mL} / \mathrm{min}^{-1}\right)$. Sensors were initially placed in the chamber having no contact with the brain slice. Once a steady-state baseline was achieved, the sensors were laid flat in direct contact with the cut surface of the cerebral cortex (Fig. 1), revealing tonic lactate release which stabilized within approximately 15 minutes. Hypoxic conditions, known to increase both lactate production due to inhibition of oxidative phosphorylation and the opening probability of connexin hemichannels, ${ }^{(13,24)}$ were induced for 2-4 minutes by replacement of oxygen in the medium with nitrogen (perfusion of the chamber with aCSF saturated with $\left.95 \% \mathrm{~N}_{2} / 5 \% \mathrm{CO}_{2}\right) .{ }^{(25,26)}$ Because detection of lactate by the biosensors requires oxygen (Supporting Fig. $\mathrm{S} 1 \mathrm{~B}),{ }^{(27)}$ the effect of hypoxia was determined by measuring the peak lactate release upon reoxygenation (Fig. 1) as described in detail previously. ${ }^{(13,27)}$ Once the baseline was restored, $\mathrm{Ca}^{2+}$-free aCSF (with the addition of $1 \mathrm{mM}$ ethylene glycol tetraacetic acid) was applied for 20 minutes as the second stimulus known to increase the opening probability of certain membrane channels, including connexin hemichannels. ${ }^{(28)}$ There is no prior evidence that $\mathrm{Ca}^{2+}$-mediated increases in mitochondrial $\mathrm{NADH}$ influence cytosolic $\mathrm{NAD}^{+} / \mathrm{NADH}$ homeostasis and therefore lactate production. ${ }^{(29)}$ These stimuli were reapplied in the presence of connexin hemichannel blockers carbenoxolone (CBX, $100 \mu \mathrm{M}$; Sigma, Poole, UK) or 5-nitro-2(3-phenylpropylamino)benzoic acid (NPPB, $200 \mu \mathrm{M}$; Sigma). CBX and NPPB have previously been shown to have no effect on lactate biosensor detection system. ${ }^{(13)}$

\section{ASSESSMENT OF HEMICHANNEL FUNCTION USING DYE LOADING}

For the assessment of hemichannel functionality (effectiveness of channel opening and closing) we used a fluorescent dye carboxyfluorescein (CBF; $376 \mathrm{Da}$ ). Connexin hemichannels are permeable to $\mathrm{CBF}$ and can act as conduits of CBF transport across the membrane in accord with the concentration gradient of the dye. Cortical slices from sham-operated, BDL, HA, and BDL-OP treated rats were exposed to normal aCSF with an addition of $\mathrm{CBF}(200 \mu \mathrm{M})$ for 9 minutes resulting in background connexin-mediated dye loading, followed by perfusion with $\mathrm{Ca}^{2+}$-free aCSF without CBF for 9 minutes resulting in $\mathrm{CBF}$ unloading. $\mathrm{Ca}^{2+}$-free aCSF with $\mathrm{CBF}$ was then applied for 4 minutes increasing the permeability of hemichannels and therefore resulting in dye loading. Hypoxic conditions (without CBF) were next applied to unload the slice and also to demonstrate bidirectional permeability of the channels to $\mathrm{CBF}{ }^{(26)}$ The same hypoxic stimulus was then reapplied in the presence of 
$\mathrm{CBF}$, resulting in dye loading. After application of each stimulus in the presence of $\mathrm{CBF}$, a further 5minute perfusion with aCSF containing $\mathrm{CBF}$ was performed, followed by a 10-minute wash with normal aCSF, enabling the channels to return to their physiological state. Images were taken using MiCAM-02 imaging system (SciMedia, Costa Mesa, CA). Using ImageJ software, regions of interest were drawn around the areas of the cerebral cortex $\left(\sim 3 \mathrm{~cm}^{2}\right)$ across layers I-III, and the mean pixel intensities for the regions were calculated. Background fluorescence was subtracted.

\section{STATISTICAL ANALYSIS}

Western blot data were normalized using the protocol of LI-COR Biosciences (Normalization Accuracy for Western Blotting), and group data were compared using two-way analysis of variance (ANOVA) with a Tukey post hoc test. Data obtained using biosensor recordings were analyzed and presented nonparametrically using box and whisker plots (Figs. 2A, 3A, 4A). For comparisons between the experimental groups, a Mann-Whitney $U$ test was applied. The peak hypoxia- or $0 \mathrm{Ca}^{2+}$-induced lactate releases are presented as changes in release from the baseline (Fig. 1). The effects of connexin blockers are presented as percent changes from the control responses recorded in the absence of the blockers, and a Wilcoxon signed rank test was applied for comparison. The $P$ values in Figs. $2 \mathrm{~B}, \mathrm{C}, 3 \mathrm{~B}, \mathrm{C}$, and $4 \mathrm{~B}, \mathrm{C}$ indicate the significance level of differences between the control responses and the responses recorded in the presence of the drugs.

Data obtained in dye-loading experiments were analyzed using two-way ANOVA (Fig. 5B, data normally distributed) followed by a Tukey post hoc test or Wilcoxon signed rank test (Fig. 5C,D, data not normally distributed) as appropriate. The biochemistry data were analyzed using one-way ANOVA. Data are reported as the mean \pm standard error of the mean. Differences with $P<0.05$ were considered to be statistically significant. Sample sizes were calculated using Gpower 3 v3.1.9.2 (http://www.gpower.hhu.de/en. html) ${ }^{(30)}$ using a 'means: Wilcoxon-Mann-Whitney test (two groups)' test, with a desired power of 90\% and a significance level of 5\%. The effect size varied between groups according to the preliminary data acquired during the study. Statistical analysis was performed using OriginPro 9.1 (OriginLab, Northampton, MA).

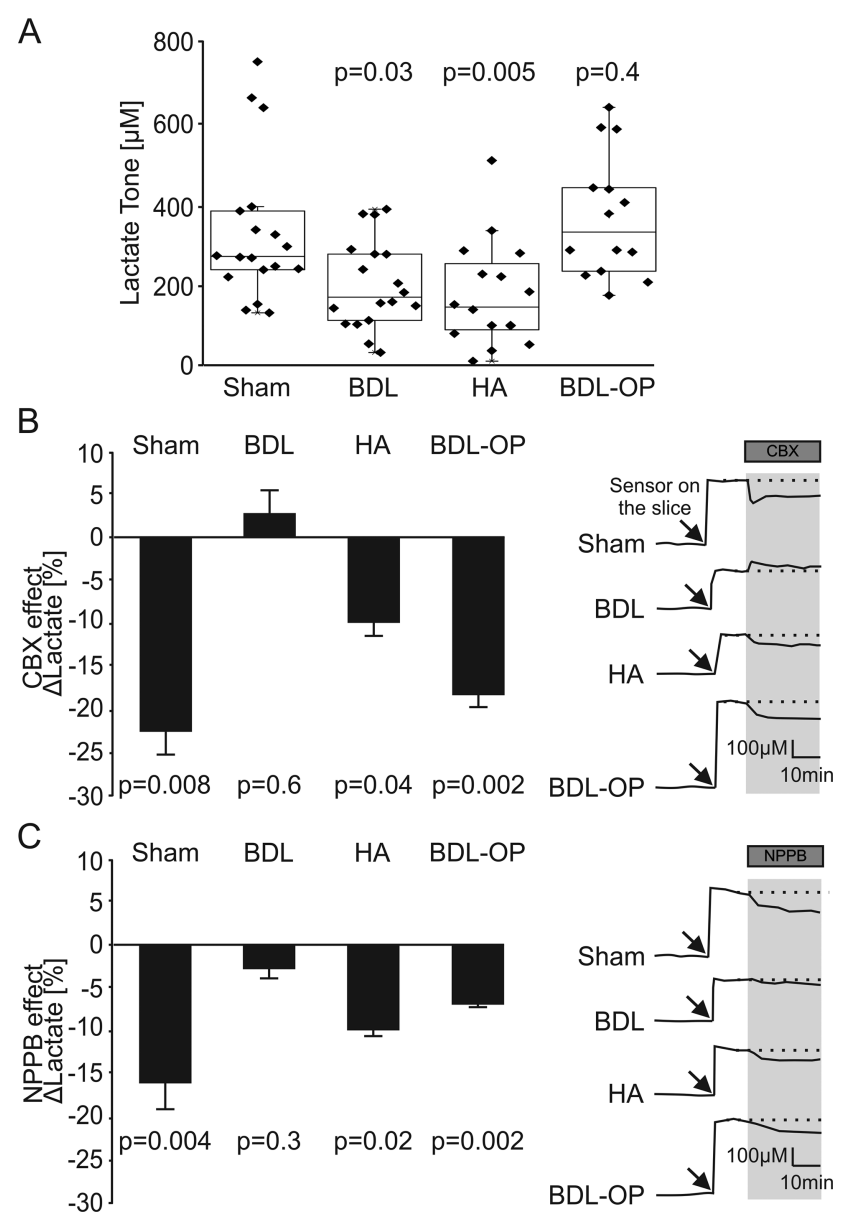

FIG. 2. HE is associated with a reduction in hemichannelmediated release of lactate in the cerebral cortex. (A) Summary data illustrating tonic release of lactate in cortical slices of shamoperated, BDL, HA, and BDL-OP treated rats. $P$ values indicate differences from the sham-operated rats. (B) Left: Summary data illustrating the effect of CBX $(100 \mu \mathrm{M})$ on tonic release of lactate (expressed as percent change from the baseline) in cortical slices of sham-operated, BDL, HA, and BDL-OP treated rats. Right: Representative recordings of lactate biosensor current showing changes in tonic release of lactate in response to CBX application. $P$ values indicate differences from the respective baseline. (C) Left: Summary data illustrating the effect of NPPB $(200 \mu \mathrm{M})$ on tonic release of lactate (expressed as percent change from the baseline) in cortical slices of sham-operated, BDL, HA, and BDL-OP treated rats. Right: Representative recordings of lactate biosensor current showing changes in tonic release of lactate in response to NPPB application. $P$ values indicate differences from the respective baseline.

\section{Results}

\section{BIOCHEMISTRY}

Plasma biochemistry and ammonia concentrations were assessed in all groups of animals (Supporting Table S1). Compared with sham surgery, BDL 
resulted in a significant increase in plasma alanine aminotransferase and bilirubin $(P<0.001)$, indicating impaired liver function, whereas albumin and total protein concentrations were significantly decreased

A

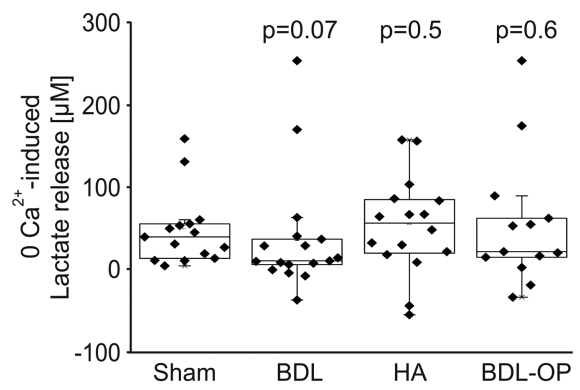

B
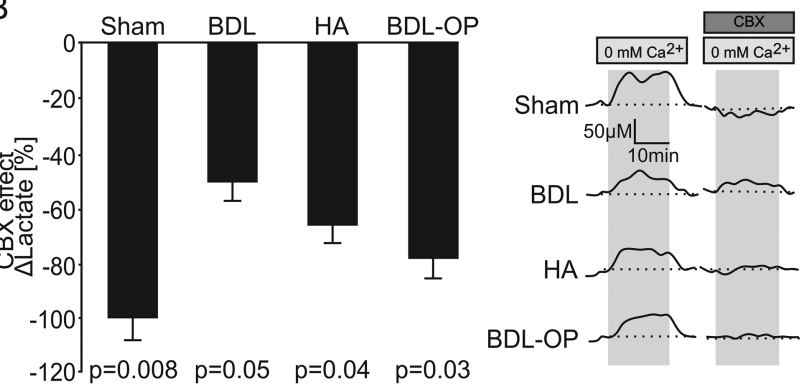

C

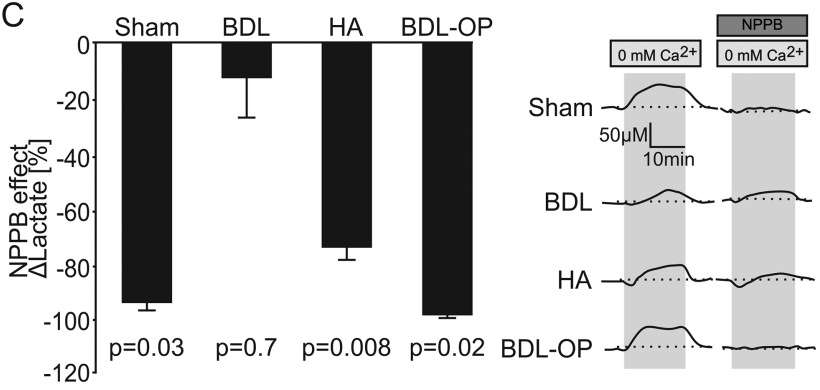

FIG. 3. Lactate release in response to lowering extracellular $\left[\mathrm{Ca}^{2+}\right]$. (A) Summary data illustrating peak changes in lactate release in response to lowering $\left[\mathrm{Ca}^{2+}\right]_{\mathrm{e}}$ in cortical slices of shamoperated, BDL, HA, and BDL-OP treated rats. $P$ values indicate differences from the responses recorded in sham-operated rats. (B) Left: Summary data illustrating the effect of CBX $(100 \mu \mathrm{M})$ on the release of lactate facilitated in response to $0\left[\mathrm{Ca}^{2+}\right]_{\mathrm{e}}$ (expressed as the percentage of the amount of lactate released in response to $0\left[\mathrm{Ca}^{2+}\right]_{e}$ in the absence of CBX) in cortical slices of shamoperated, BDL, HA, and BDL-OP treated rats. Right: Representative recordings of lactate biosensor current showing the effect of CBX on $0\left[\mathrm{Ca}^{2+}\right]_{e}-$ induced release of lactate. $P$ values indicate differences between the responses recorded in the absence and presence of CBX. (C) Left: Summary data illustrating the effect of NPPB $(200 \mu \mathrm{M})$ on the release of lactate facilitated in response to $0\left[\mathrm{Ca}^{2+}\right]_{\mathrm{e}}$ (expressed as the percentage of the amount of lactate released in response to $0\left[\mathrm{Ca}^{2+}\right]_{\mathrm{e}}$ in the absence of NPPB) in cortical slices of sham-operated, BDL, HA, and BDL-OP treated rats. Right: Representative recordings of lactate biosensor current showing the effect of NPPB on $0\left[\mathrm{Ca}^{2+}\right]_{\mathrm{e}}$-induced release of lactate. $P$ values indicate differences between the responses recorded in the absence and presence of NPPB.

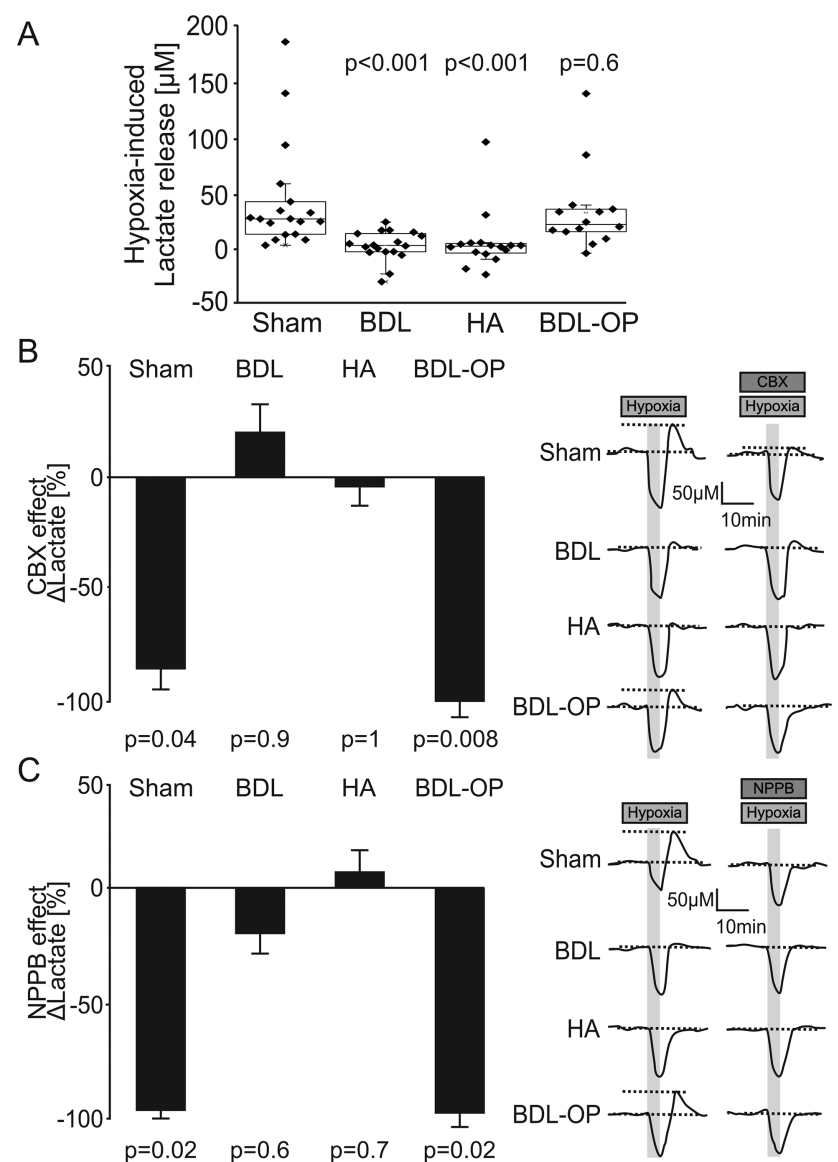

FIG. 4. HE is associated with a reduction of hypoxia-induced release of lactate. (A) Summary data illustrating peak changes in lactate release in response to hypoxia $(\mathrm{aCSF}$ saturated with $95 \% \mathrm{~N}_{2} / 5 \% \mathrm{CO}_{2}$ ) in cortical slices of sham-operated, BDL, $\mathrm{HA}$, and BDL-OP treated rats. $P$ values indicate differences from the responses recorded in sham-operated rats. (B) Left: Summary data illustrating the effect of CBX $(100 \mu \mathrm{M})$ on the release of lactate facilitated in response to hypoxia (expressed as the percentage of the amount of lactate released in response to hypoxia in the absence of CBX) in cortical slices of shamoperated, BDL, HA, and BDL-OP treated rats. Right: Representative recordings of lactate biosensor current showing the effect of CBX on hypoxia-induced release of lactate. Decrease in $\mathrm{O}_{2}$ availability reduces biosensor current followed by a positive signal upon reoxygenation, which is used to estimate hypoxia-induced lactate release. $P$ values indicate differences between the responses recorded in the absence and presence of CBX. (C) Left: Summary data illustrating the effect of NPPB $(200 \mu \mathrm{M})$ on the release of lactate facilitated in response to hypoxia (expressed as the percentage of the amount of lactate released in response to hypoxia in the absence of NPPB) in cortical slices of sham-operated, BDL, $\mathrm{HA}$, and BDL-OP treated rats. Right: Representative recordings of lactate biosensor current showing the effect of NPPB on the hypoxia-induced release of lactate. $P$ values indicate differences between the responses recorded in the absence and presence of NPPB. 
$(P<0.001)$. Treatment of BDL rats with OP had no effect on these parameters. Rats fed an HA diet had plasma biochemistry similar to that of control rats.

Plasma ammonia concentrations were significantly higher in BDL and HA rats when compared with sham-operated rats $(P<0.001)$. Treatment of $\mathrm{BDL}$ rats with OP lowered plasma ammonia concentration, which was similar to that measured in sham-operated rats $(P=0.3)$ (Supporting Table S1).

\section{RELEASE OF LACTATE IN THE CEREBRAL CORTEX IN ANIMAL MODELS OF HE}

In cortical slices of sham-operated rats, enzymatic amperometric biosensors detected tonic lactate efflux of $335 \pm 10 \mu \mathrm{M}(\mathrm{n}=18)$. Recordings from cortical slices of BDL and HA rats showed lower tonic release of lactate of $203 \pm 6 \mu \mathrm{M}(P=0.03, \mathrm{n}=18)$ and $178 \pm$ $8 \mu \mathrm{M} \quad(P=0.005, \mathrm{n}=16)$, respectively (Fig. $2 \mathrm{~A})$. Increasing the permeability of connexin hemichannels by lowering $\left[\mathrm{Ca}^{2+}\right]_{\mathrm{e}}$ triggered similar increases in the release of lactate in sham-operated rats (by $43 \pm 3 \mu \mathrm{M}$, $\mathrm{n}=15$ ), BDL rats (by $38 \pm 4 \mu \mathrm{M} ; P=0.07, \mathrm{n}=17$ ), and $\mathrm{HA}$ rats (by $54 \pm 4 \mu \mathrm{M} ; P=0.5, \mathrm{n}=16$ ) (Fig. $3 \mathrm{~A})$. Hypoxia facilitated the release of lactate in cortical slices of sham-operated rats $(43 \pm 3 \mu \mathrm{M}, \mathrm{n}=18)$ but had no effect on lactate release in slices of BDL rats $(1 \pm 0.8 \mu \mathrm{M} ; P<0.001, \mathrm{n}=18)$ or HA rats $(5 \pm$ $2 \mu \mathrm{M} ; P<0.001, \mathrm{n}=16$ ) (Fig. 4A). These results demonstrated impaired tonic and hypoxia-induced release of lactate in both animal models of $\mathrm{HE}$.

\section{AMMONIA LOWERING TREATMENT RESTORES CORTICAL LACTATE RELEASE}

OP treatment had been shown to decrease systemic and brain ammonia concentrations in BDL rats. ${ }^{(20)}$ We next found that in our experiments, OP treatment of BDL rats restored tonic $(374 \pm 11 \mu \mathrm{M} ; P=0.4$, $\mathrm{n}=14)$ and hypoxia-induced $(32 \pm 3 \mu \mathrm{M} ; P=0.6$, $\mathrm{n}=14$ ) lactate release similar to that recorded in cortical slices of sham-operated rats (Figs. 2A, 3A, and 4A). Direct application of OP on cortical slices of sham-operated and BDL rats had no effect on lactate release (Supporting Fig. S1D). These results suggest that high ammonia levels are responsible for the reduction in lactate release in the cerebral cortex of BDL rats.

\section{IMPAIRED HEMICHANNEL FUNCTION UNDERLIES REDUCED CORTICAL LACTATE RELEASE IN ANIMAL MODELS OF HE}

In cortical slices of sham-operated rats, application of hemichannel blockers CBX $(n=9)$ or NPPB $(n=9)$ resulted in a significant reduction in lactate release (Fig. 2B,C). Hemichannel blockade had no effect on lactate tone in cortical slices of BDL rats (Fig. 2B,C). However, hemichannel blockade had an effect on lactate release recorded in cortical slices of $H A$ rats (CBX: $n=10$; NPPB: $n=8$ ) (Fig. 2B,C). $\mathrm{CBX}$ and NPPB reduced lactate tone in cortical slices of BDL rats treated with OP $(\mathrm{CBX}, \mathrm{n}=10$; NPPB, $\mathrm{n}=10$ ) (Fig. 2B,C).

In cortical slices of sham-operated $(\mathrm{CBX}, \mathrm{n}=8$; $\mathrm{NPPB}, \mathrm{n}=6)$ and $\mathrm{HA}$ rats $(\mathrm{CBX}, \mathrm{n}=10$; NPPB, $\mathrm{n}=8$ ), hemichannel blockade using CBX or NPPB abolished or significantly reduced the amount of lactate release facilitated in $0 \mathrm{Ca}^{2+}$ conditions (Fig. 3B,C). A smaller effect of hemichannel blockade on $0 \mathrm{Ca}^{2+}$ induced release of lactate was observed in cortical slices of $\mathrm{BDL}$ rats (CBX, $\mathrm{n}=11 ; \mathrm{NPPB}, \mathrm{n}=9$ ) (Fig. $3 B, C)$. In conditions of OP treatment, when tonic lactate release was restored in cortical slices of BDL rats, CBX $(n=7)$ and NPPB $(n=7)$ abolished the release of lactate facilitated by $0 \mathrm{Ca}^{2+}$, an effect similar to that observed after application of hemichannel blockers in cortical slices of sham-operated rats (Fig. 3B,C).

Hypoxia-induced release of lactate recorded in cortical slices of sham-operated rats was abolished or dramatically reduced by connexins blockade $(\mathrm{CBX}, \mathrm{n}=9$; NPPB, $n=7$ ) (Fig. 4B,C). CBX or NPPB had no significant effect on the release of lactate induced by hypoxia in cortical slices of BDL (CBX, $n=13$; NPPB, $n=9$ ) or HA rats (CBX, $n=10$; NPPB, $n=12$ ) (Fig. $4 \mathrm{~B}, \mathrm{C})$. In cortical slices of OP-treated BDL rats, the effects of CBX and NPPB were restored. CBX $(n=8)$ and NPPB $(n=7)$ effectively abolished hypoxiainduced lactate release in cortical slices of BDL rats treated with OP (Fig. 4B,C).

Application of the monocarboxylate transporter (MCT) blocker $\alpha$-cyano-4-hydroxycinnamic acid (4CIN) had no significant effect on tonic release of lactate in cortical slices of sham-operated $(n=5)$ and BDL rats $(n=5)$ (Supporting Fig. S2A,B). Hypoxiainduced lactate release recorded in sham-operated rats $(n=5)$ was significantly reduced by $4-C I N$, as 


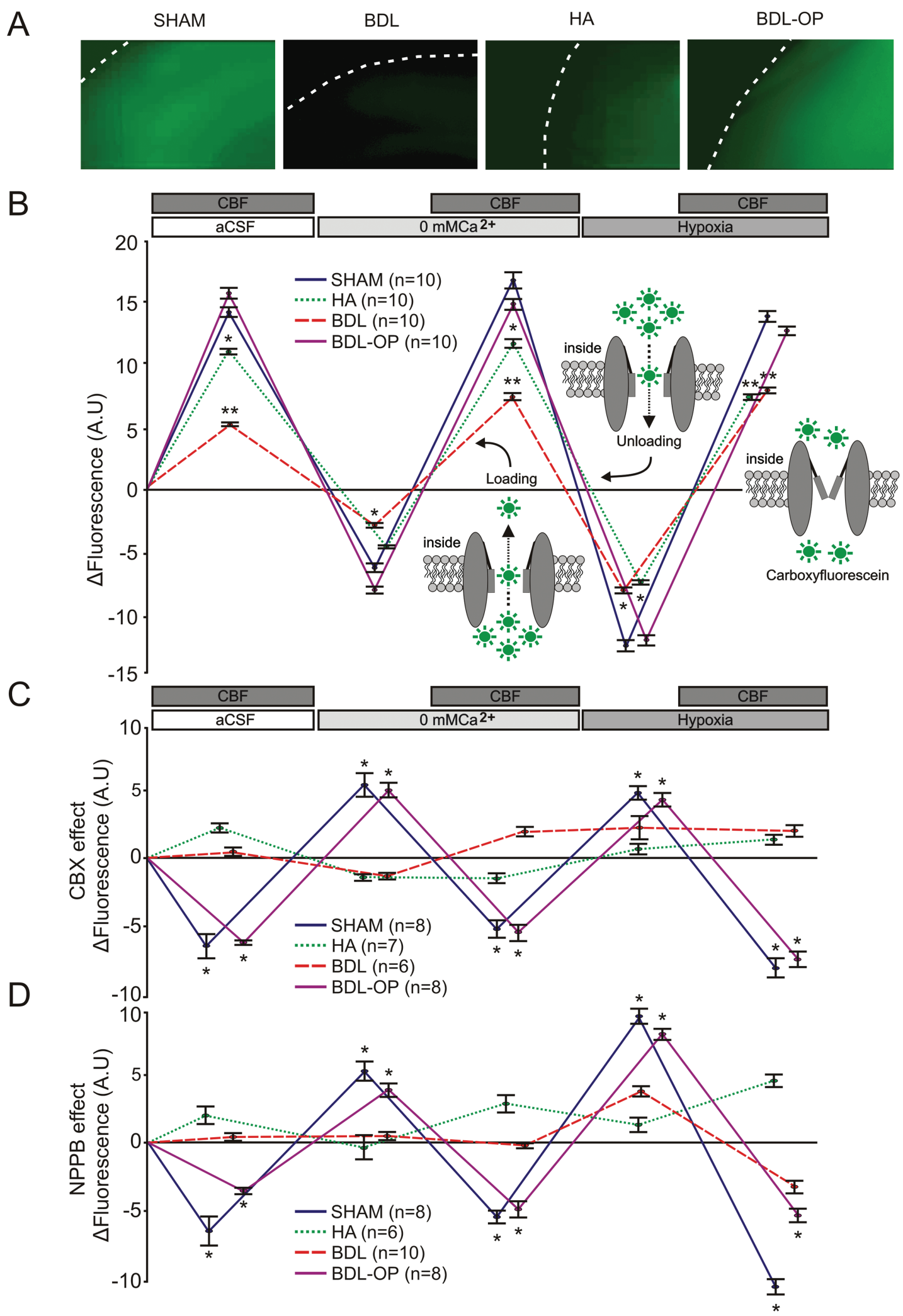

FIG. 5 
reported previously. ${ }^{(13)}$ In cortical slices of BDL rats $(n=5)$ the effect of 4-CIN on hypoxia-induced release of lactate was found to be smaller (Supporting Fig. S2A,B).

These results demonstrate that connexin hemichannel blockade has no effect on the release of lactate in the cerebral cortex of BDL rats. This implies that the function of hemichannels, which may act as conduits of lactate release, ${ }^{(13)}$ is already compromised in the brains of these rats. These data also suggest that cortical hemichannel dysfunction in the BDL rats is likely to be due to the actions of ammonia.

\section{CORTICAL HEMICHANNEL- MEDIATED DYE LOADING IN ANIMAL MODELS OF HE}

Next, membrane channel-mediated dye loading experiments were performed in cortical slices to confirm hemichannel dysfunction in HE. In cortical slices of sham-operated rats $(\mathrm{n}=10)$, significant background loading (14.3 $\pm 0.4 \mathrm{AU})$ was observed in control conditions when slices were perfused with aCSF containing $\mathrm{CBF}$ (Fig. 5A,B). Increasing the permeability of hemichannels by lowering $\left[\mathrm{Ca}^{2+}\right]_{\mathrm{e}}$ in the absence of $\mathrm{CBF}$ reduced slice fluorescence by $6.4 \pm 0.2 \mathrm{AU}$. The same stimulus applied in the presence of CBF significantly increased fluorescence by $16.9 \pm 0.6$ AU. Hypoxia-induced opening of hemichannels in the absence of $\mathrm{CBF}$ resulted in dye unloading with fluorescence decreasing by $12.7 \pm 0.4 \mathrm{AU}$. The addition of $\mathrm{CBF}$ in conditions of hypoxia increased slice fluorescence by $13.9 \pm 0.4 \mathrm{AU}$ (Fig. 5B).

Cortical slices from HA rats $(n=10)$ displayed background loading (Fig. 5A,B) and $0 \mathrm{Ca}^{2+}$-induced unloading of $11.2 \pm 0.2 \mathrm{AU}(P=0.04)$ and $4.7 \pm 0.08$
AU $(P=0.4)$, respectively (Fig. 5B). The addition of $\mathrm{CBF}$ in $0 \mathrm{Ca}^{2+}$ conditions increased fluorescence by $11.6 \pm 0.2 \mathrm{AU}(P=0.004)$, similar to that observed in slices of sham-operated rats. However, the effect of hypoxia was significantly reduced in slices of HA rats (unloading by $7.6 \pm 0.1 \mathrm{AU}, P<0.001$; loading by $7.8 \pm 0.2 \mathrm{AU}, P<0.001$ ) (Fig. 5B).

In cortical slices of BDL rats $(n=10)$, the efficacy of $\mathrm{CBF}$ dye loading and unloading was markedly reduced under all conditions (Fig. 5A,B). In cortical slices of BDL rats treated with OP, hemichannelmediated $\mathrm{CBF}$ dye loading and unloading were similar to that observed in slices of sham-operated rats (Fig. $5 \mathrm{~A}, \mathrm{~B})$.

Hemichannel blockade with CBX or NPPB markedly reduced CBF dye loading and unloading in cortical slices of sham-operated rats (Fig. 5C-D). In cortical slices of BDL and HA rats, CBX and NPPB had no significant effect on dye loading and unloading (Fig. 5C,D). In cortical slices of OP-treated BDL rats, the effects of CBX and NPPB on CBF dye loading and unloading were similar to that observed in shamoperated rats. (Fig. 5C,D). Figure 5C,D illustrates changes in fluorescence ( $\Delta$ Fluorescence) following application of the hemichannel blockers, compared with the respective changes in fluorescence recorded in the absence of blockers in slices of the same animal. Negative values show decreases in fluorescence, and positive values illustrate higher fluorescence levels compared with controls.

These data show that the background activity and stimuli-evoked opening and closure of connexin hemichannels is impaired in BDL rats. The efficacy of CBF dye loading was restored by $\mathrm{OP}$ treatment, suggesting that the actions of ammonia are responsible for cortical hemichannel dysfunction in $\mathrm{HE}$.

FIG. 5. Impaired hemichannel-mediated dye loading reveals cortical hemichannel dysfunction in HE. (A) Representative images of background loading with $\mathrm{CBF}$ dye in cortical slices of sham-operated, BDL, HA, and BDL-OP treated rats. White dashed lines depict the edge of each cortical slice. (B) Fluorescence intensity changes in cortical slices of sham-operated, BDL, HA, and BDL-OP treated rats in response to $0\left[\mathrm{Ca}^{2+}\right]_{\mathrm{e}}$ and hypoxia in the absence and presence of $\mathrm{CBF}$ in the medium. Application of $0\left[\mathrm{Ca}^{2+}\right]_{\mathrm{e}}$ aCSF or hypoxia in the presence of CBF resulted in dye loading and an increase in fluorescence, whereas application of these stimuli in the absence of CBF resulted in dye unloading and a decrease in fluorescence. Insets: Schematic drawings of connexin hemichannel mediated dye loading and unloading. ${ }^{*} P<0.05,{ }^{* *} P<0.001$ versus sham-operated animals. (C) Summary data illustrating the effect of CBX $(100 \mu \mathrm{M})$ on fluorescence intensity changes ( $\Delta$ Fluorescence) in cortical slices of sham-operated, BDL, HA, and BDL-OP treated rats induced by $0\left[\mathrm{Ca}^{2+}\right]_{\mathrm{e}}$ and hypoxia in the absence and presence of CBF in the medium. The data are presented as differences in fluorescence after CBX application compared with the respective fluorescence recorded in the absence of $\mathrm{CBX}$. ${ }^{*} P<0.05$ for the effect of CBX on CBF loading and unloading. (D) Summary data illustrating the effect of NPPB (200 $\mu \mathrm{M})$ on fluorescence intensity changes $(\Delta$ Fluorescence $)$ in cortical slices of sham-operated, BDL, HA, and BDL-OP treated rats induced by $0\left[\mathrm{Ca}^{2+}\right]_{\mathrm{e}}$ and hypoxia in the absence and presence of CBF in the medium. The data are presented as differences in fluorescence after NPPB application compared with the respective fluorescence recorded in the absence of NPPB. ${ }^{*} P<0.05$ for the effect of NPPB on CBF loading and unloading. 

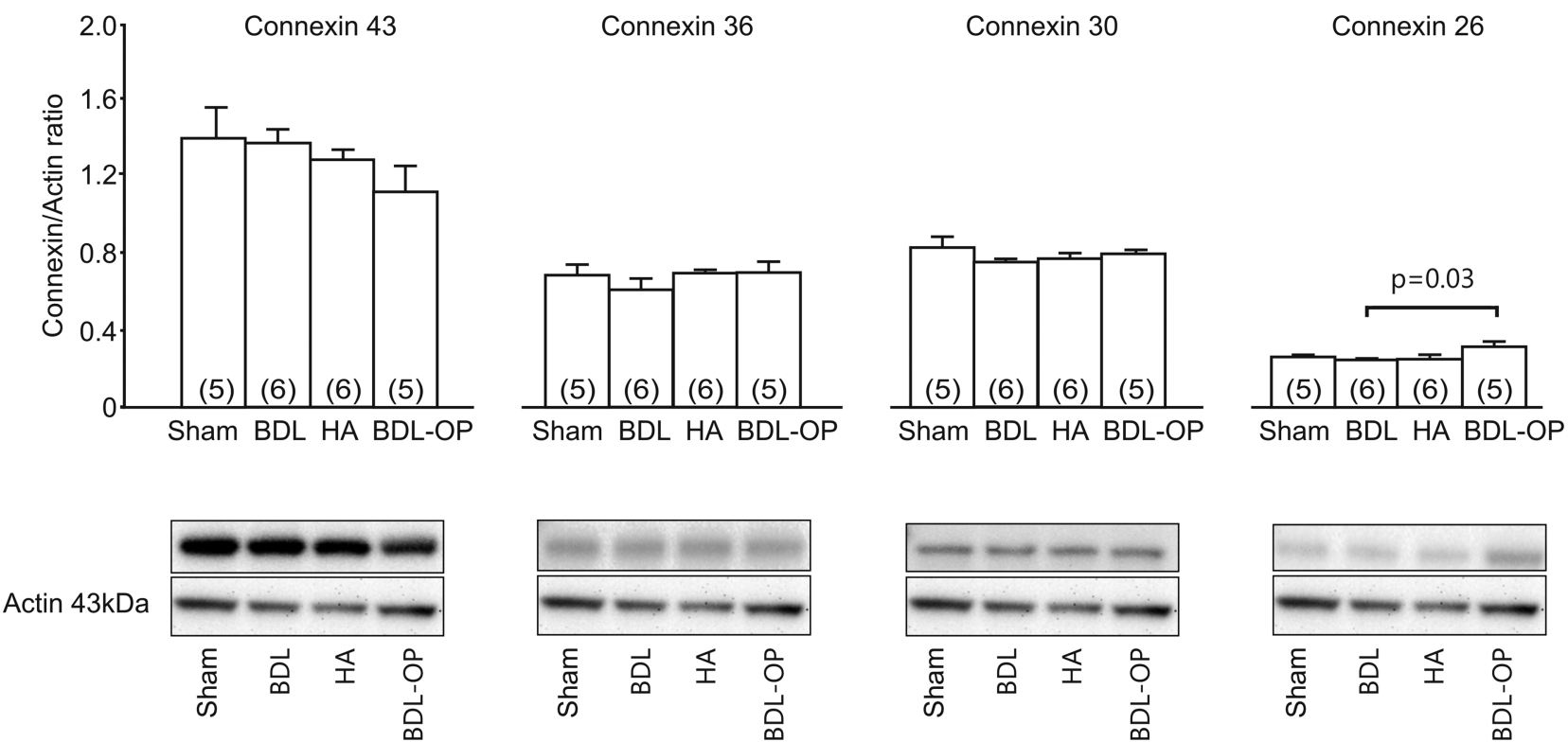

FIG. 6. Connexin expression in the cortex is not affected in HE. Top: Summary data illustrating means \pm standard error of the densitometry of connexin-43, connexin-36, connexin-30, and connexin-26 protein expression normalized to the expression of actin in cell lysates of the cerebral cortices of sham-operated, BDL, HA, and BDL-OP treated rats. Bottom: Representative immunoblots showing connexin-43, connexin-36, connexin-30, and connexin-26 protein expression in cerebral cortices of sham-operated, BDL, HA, and BDL-OP treated rats. The $P$ value indicates the difference in expression level between the BDL and BDL-OP treated groups.

\section{CORTICAL CONNEXIN EXPRESSION IN ANIMAL MODELS OF HE}

We next evaluated cortical expression of main astrocytic and neuronal connexins in animal models of $\mathrm{HE}$ used in this study. Western blotting was performed on proteins extracted from the cerebral cortices of shamoperated, BDL, HA, and BDL-OP treated rats (Fig. $6)$. No differences in cortical connexin-43, connexin36, or connexin-30 expression was observed between sham-operated, BDL, HA, and OP-treated BDL rats. A small increase in connexin-26 expression $(P=0.03)$ was observed in BDL-OP treated rats compared with $\mathrm{BDL}$ rats (Fig. 6). Expression of MCT-1 was similar in all experimental groups (Supporting Fig. S2C).

\section{Discussion}

Brain information processing requires a constant and sufficient supply of oxygen and metabolic substrates. Astrocytes represent an important source of lactate, which contributes to the extracellular pool of readily available metabolic substrates taken up by neurons to fuel their activity. ${ }^{(31)}$ Although it was thought previously that lactate transport across the cell membranes was achieved solely through the operation of MCTs, a recent study ${ }^{(13)}$ demonstrated that connexin hemichannels are equally important conduits of lactate release.

In animal models of and patients with ALF, an increase in brain lactate concentration has been reported. ${ }^{(32)}$ Concentrations of lactate in the cerebrospinal fluid were also found to be elevated in patients with cirrhosis, but only in severe cases of HE. ${ }^{33)}$ The BDL and HA rats used in our experiments are models of minimal HE. ${ }^{(34)}$ In contrast to the existing evidence suggesting that brain lactate concentrations are increased in $\mathrm{ALF}{ }^{(35)}$ our experiments demonstrated that the development of $\mathrm{HE}$ in rats is associated with a significant reduction in tonic and stimulated release of lactate in the cerebral cortex. Blockade of connexin hemichannels was found to be effective in reducing lactate release in sham-operated and HA rats but was ineffective in BDL rats, suggesting that the reduction in hemichannel-mediated lactate release in BDL rats is due to a combination of pathological factors (e.g., inflammation, oxidative stress).

Increased lactate production by astrocytes appears to be essential for the recovery of synaptic function during reoxygenation after hypoxia. ${ }^{(36)} \mathrm{We}$ found that 
hypoxia-induced lactate release was significantly reduced in the cerebral cortex in BDL and $\mathrm{HA}$ rats compared with control rats, and was unaffected by the connexin blockers, indicating hemichannel dysfunction. The observed decrease in extracellular lactate is likely due to impaired release from astrocytes, although increased neuronal activity and therefore lactate consumption cannot be excluded. The results reported by Bosoi et al. ${ }^{(37)}$ showing higher total brain lactate in BDL rats using NMR spectroscopy seem to contradict our data. Bosoi et al. suggested that increased lactate contributes to the pathogenesis of brain edema (cytotoxic) and may imply that the observed increase in total brain lactate is due to its intracellular accumulation. If the rate of lactate production and glymphatic clearance $^{(38)}$ are not affected, intracellular retention of lactate would explain higher concentration of this metabolite as measured by NMR spectroscopy ${ }^{(39)}$ and would be in full agreement with our data showing impairment of hemichannel-mediated release in HE.

High concentration of ammonia can potentially generate significant $\mathrm{pH}$ changes, which can have various effects on many $\mathrm{pH}$ sensitive membrane channels, including hemichannels. The $\mathrm{pH}$ sensitivity of hemichannels is known as the chemical gate, which is the phenomenon of hemichannel blockade when intracellular $\mathrm{pH}\left(\mathrm{pH}_{\mathrm{i}}\right)$ decreases. ${ }^{(39)}$ This provides one potential mechanism that may be responsible for impaired hemichannel-mediated lactate release in $\mathrm{HE}$.

We also examined hemichannel functionality using the dye-loading method. Some differences between the data obtained using biosensor recordings and this technique could be due to the fact that $\mathrm{CBF}$ is not identical to the molecular structure and size of lactate. Additionally, lactate could be released through specific connexin hemichannels, whereas $\mathrm{CBF}$ is small enough to pass through the majority of hemichannels expressed by both astrocytes and neurons. Whereas hypoxia may predominantly affect astroglial hemichannels, the rest of the experimental conditions are not "cell specific" and conclusions regarding specific cell types involved cannot be drawn from the results obtained in dye-loading experiments.

The experimental stimuli used $\left(0 \mathrm{Ca}^{2+}\right.$ and hypoxia) are known to increase the permeability of hemichannels, possibly by affecting various protein bonds resulting in conformational changes. ${ }^{(28)}$ Dye-loading experiments clearly demonstrated significant reduction in fluorescent dye uptake and release in cortical slices of BDL and HA rats compared with sham-operated rats (the differences were more profound when hypoxia was used as a stimulus), suggesting reduced bidirectional permeability of hemichannels in these animal models of HE. Hemichannel blockade had little effect on fluorescent dye uptake in BDL and HA rats, providing additional evidence that the function of these channels is already compromised. Ammonium ions may cause structural alterations to the connexin proteins by interacting with various amino acid side chains, which could affect gating of the channel. However, because hemichannels have a relatively short life cycle and are recycled frequently, the observed changes in hemichannel functionality appear to be reversible with OP treatment.

In chronic liver disease, hyperammonemia is believed to impair mitochondrial function and induce astroglial dysfunction, which is associated with altered neurotransmitter recycling leading to neuronal damage. ${ }^{(40)}$ Ammonia may also interfere with cell energy metabolism in several ways. There is recent evidence that in astrocytes, ammonia may acutely divert the flux of pyruvate to lactate production, contributing to the net aerobic lactate production. ${ }^{(7)}$ However, the effects of chronic ammonia exposure on astrocytes are unknown. We investigated the role of ammonia by treating BDL rats with $\mathrm{OP}$, a drug known to lower systemic and brain ammonia. (20) OP treatment improved the neurochemical phenotype of BDL rats by restoring the tonic and stimulated hemichannelmediated lactate release. Furthermore, hemichannel blockade became effective after OP treatment, suggesting that ammonia is indeed responsible for hemichannel dysfunction observed in this model.

Cytotoxic brain edema observed in BDL rats is attenuated by ammonia-lowering treatments such as the one used in the present study. ${ }^{(20,41)}$ The effect of cell swelling on hemichannel function is poorly understood. Ye et al. ${ }^{(42)}$ showed that astrocytes obtained from connexin-43 knockout animals developed cell swelling as efficiently as wild-type animals when exposed to a hypotonic solution, suggesting that hemichannels do not play a significant role in this process despite evidence to the contrary. ${ }^{(43)}$

We also examined the expression profile of key astroglial and neuronal connexins in the animal models of $\mathrm{HE}$ used in this study. No significant differences in connexin hemichannel protein expression profile were observed, suggesting that $\mathrm{HE}$ is associated with altered hemichannel function but not with changes in connexin expression. The up-regulated expression of connexin-26 observed in BDL-OP treated rats is not prominent enough to explain the marked improvement observed in the lactate measurements and dye- 
loading experiments. Additionally, we did not observe any changes in the expression of the main astroglial lactate transporter MCT-1 in the models of $\mathrm{HE}$ used in this study. No effect of the MCT blocker 4-CIN on lactate release was observed in cortical slices of sham-operated or BDL rats. Hypoxia-induced lactate release in sham-operated rats was found to be significantly reduced by the application of 4-CIN, as reported previously. ${ }^{(13)}$

Depletion of lactate as one of the key readily available metabolic substrates may have important neurological consequences, particularly in patients with advanced cirrhosis given the fact that these patients display evidence of cerebral vasoconstriction, ${ }^{(44)}$ which is associated with impaired cerebral autoregulation, an important mechanism that ensures constant cerebral blood flow. ${ }^{(45)}$ The clinical consequences of this may be relevant during liver transplantation, where further reductions in cerebral blood flow have been observed during the anhepatic phase of transplantation and may contribute to posttransplantation neurologic dysfunction. ${ }^{(46)}$ Evidence for critical reduction in cerebral oxygenation was obtained in the majority of patients with acute-on-chronic liver failure who had poor neurologic outcome, supporting the hypothesis that the brain energy metabolism is critically compromised in cirrhosis and that further perturbations as demonstrated in the present study may be clinically deleterious. ${ }^{(47)}$ Our data indicating an impaired hemichannel-mediated lactate release during tissue hypoxia, in combination with these observations, could help explain the severe neurological manifestations in patients with $\mathrm{HE}$. Because ammonia is central in causing this dysfunction, the potential clinical implications involve the use of ammonia-lowering treatments as a main therapeutic strategy, as well as attempts to increase cerebral oxygenation to preserve neuronal function.

In conclusion, the results of the present study suggest that $\mathrm{HE}$ is associated with central nervous system hemichannel dysfunction, with ammonia playing a key role. The data provide evidence of a potential neuronal energy deficit due to impaired hemichannel-mediated lactate transport between astrocytes and neurons as a possible mechanism underlying the pathogenesis of $\mathrm{HE}$.

\section{REFERENCES}

1) Ciecko-Michalska I, Szczepanek M, Slowik A, Mach T. Pathogenesis of hepatic encephalopathy. Gastroenterol Res Pract 2012; 2012:642108.
2) Prakash R, Mullen KD. Mechanisms, diagnosis and management of hepatic encephalopathy. Nat Rev Gastroenterol Hepatol 2010; 7:515-525.

3) Jalan R, Olde Damink SW, Hayes PC, Deutz NE, Lee A. Pathogenesis of intracranial hypertension in acute liver failure: inflammation, ammonia and cerebral blood flow. J Hepatol 2004;41: 613-620.

4) Bosoi CR, Rose CF. Oxidative stress: a systemic factor implicated in the pathogenesis of hepatic encephalopathy. Metab Brain Dis 2013;28:175-178.

5) Rao KV, Norenberg MD. Cerebral energy metabolism in hepatic encephalopathy and hyperammonemia. Metab Brain Dis 2001; 16:67-78.

6) Shawcross D, Jalan R. The pathophysiologic basis of hepatic encephalopathy: central role for ammonia and inflammation. Cell Mol Life Sci 2005;62:2295-2304.

7) Lerchundi R, Fernandez-Moncada I, Contreras-Baeza Y, SoteloHitschfeld T, Machler P, Wyss MT, et al. NH4 + triggers the release of astrocytic lactate via mitochondrial pyruvate shunting. Proc Natl Acad Sci U S A 2015;112:11090-11095.

8) Rose C, Ytrebo LM, Davies NA, Sen S, Nedredal GI, Belanger $\mathrm{M}$, et al. Association of reduced extracellular brain ammonia, lactate, and intracranial pressure in pigs with acute liver failure. Hepatology 2007;46:1883-1892.

9) Bennett MV, Contreras JE, Bukauskas FF, Saez JC. New roles for astrocytes: gap junction hemichannels have something to communicate. Trends Neurosci 2003;26:610-617.

10) Contreras JE, Saez JC, Bukauskas FF, Bennett MV. Gating and regulation of connexin $43(\mathrm{C} x 43)$ hemichannels. Proc Natl Acad Sci U S A 2003;100:11388-11393.

11) Bukauskas FF, Peracchia C. Two distinct gating mechanisms in gap junction channels: $\mathrm{CO} 2$-sensitive and voltage-sensitive. Biophys J 1997;72:2137-2142.

12) Giaume C, Koulakoff A, Roux L, Holcman D, Rouach N. Astroglial networks: a step further in neuroglial and gliovascular interactions. Nat Rev Neurosci 2010;11:87-99.

13) Karagiannis A, Sylantyev S, Hadjihambi A, Hosford PS, Kasparov S, Gourine AV. Hemichannel-mediated release of lactate. J Cereb Blood Flow Metab 2016;36:1202-1211.

14) Jalan R, Wright G, Davies NA, Hodges SJ. L-Ornithine phenylacetate $(\mathrm{OP})$ : a novel treatment for hyperammonemia and hepatic encephalopathy. Med Hypotheses 2007;69:10641069.

15) Oria M, Romero-Gimenez J, Arranz JA, Riudor E, Raguer N, Cordoba J. Ornithine phenylacetate prevents disturbances of motor-evoked potentials induced by intestinal blood in rats with portacaval anastomosis. J Hepatol 2012;56:109-114.

16) Ytrebo LM, Kristiansen RG, Maehre H, Fuskevag OM, Kalstad $\mathrm{T}$, Revhaug A, et al. L-ornithine phenylacetate attenuates increased arterial and extracellular brain ammonia and prevents intracranial hypertension in pigs with acute liver failure. Hepatology 2009;50:165-174.

17) Harry D, Anand R, Holt S, Davies S, Marley R, Fernando B, et al. Increased sensitivity to endotoxemia in the bile duct-ligated cirrhotic Rat. Hepatology 1999;30:1198-1205.

18) Riggs A. The amino acid composition of some mammalian hemoglobins: mouse, guinea pig, and elephant. J Biol Chem 1963;238:2983-2987.

19) Balata S, Olde Damink SW, Ferguson K, Marshall I, Hayes PC, Deutz NE, Williams R, Wardlaw J, Jalan R. Induced hyperammonemia alters neuropsychology, brain MR spectroscopy 
and magnetization transfer in cirrhosis. Hepatology 2003;37: 931-939.

20) Davies NA, Wright G, Ytrebo LM, Stadlbauer V, Fuskevag $\mathrm{OM}$, Zwingmann $\mathrm{C}$, et al. L-ornithine and phenylacetate synergistically produce sustained reduction in ammonia and brain water in cirrhotic rats. Hepatology 2009;50:155-164.

21) Llaudet E, Botting NP, Crayston JA, Dale N. A three-enzyme microelectrode sensor for detecting purine release from central nervous system. Biosens Bioelectron 2003;18:43-52.

22) Tian F, Gourine AV, Huckstepp RT, Dale N. A microelectrode biosensor for real time monitoring of L-glutamate release. Anal Chim Acta 2009;645:86-91.

23) Gourine AV, Llaudet E, Thomas T, Dale N, Spyer KM. Adenosine release in nucleus tractus solitarii does not appear to mediate hypoxia-induced respiratory depression in rats. J Physiol 2002;544:161-170.

24) Orellana JA, Hernandez DE, Ezan P, Velarde V, Bennett MV, Giaume $\mathrm{C}$, et al. Hypoxia in high glucose followed by reoxygenation in normal glucose reduces the viability of cortical astrocytes through increased permeability of connexin 43 hemichannels. Glia 2010;58:329-343.

25) Huckstepp RT, id Bihi R, Eason R, Spyer KM, Dicke N, Willecke $\mathrm{K}$, et al. Connexin hemichannel-mediated $\mathrm{CO}_{2}$-dependent release of ATP in the medulla oblongata contributes to central respiratory chemosensitivity. J Physiol 2010;588:3901-3920.

26) Meigh L, Greenhalgh SA, Rodgers TL, Cann MJ, Roper DI, Dale N. CO(2)directly modulates connexin 26 by formation of carbamate bridges between subunits. Elife 2013;2:e1213.

27) Turovsky E, Karagiannis A, Abdala AP, Gourine AV. Impaired $\mathrm{CO} 2$ sensitivity of astrocytes in a mouse model of Rett syndrome. J Physiol 2015;593:3159-3168.

28) Muller DJ, Hand GM, Engel A, Sosinsky GE. Conformational changes in surface structures of isolated connexin 26 gap junctions. EMBO J 2002;21:3598-3607.

29) Marcu R, Wiczer BM, Neeley CK, Hawkins BJ. Mitochondrial matrix $\mathrm{Ca}(2)(+)$ accumulation regulates cytosolic $\mathrm{NAD}(+)$ / $\mathrm{NADH}$ metabolism, protein acetylation, and sirtuin expression. Mol Cell Biol 2014;34:2890-2902.

30) Faul F, Erdfelder E, Buchner A, Lang AG. Statistical power analyses using $G^{*}$ Power 3.1: tests for correlation and regression analyses. Behav Res Methods 2009;41:1149-1160.

31) Pellerin L, Magistretti PJ. Glutamate uptake into astrocytes stimulates aerobic glycolysis: a mechanism coupling neuronal activity to glucose utilization. Proc Natl Acad Sci U S A 1994;91:10625-10629.

32) Rose CF. Increase brain lactate in hepatic encephalopathy: cause or consequence? Neurochem Int 2010;57:389-394.

33) Yao H, Sadoshima S, Fujii K, Kusuda K, Ishitsuka T, Tamaki $\mathrm{K}$, et al. Cerebrospinal fluid lactate in patients with hepatic encephalopathy. Eur Neurol 1987;27:182-187.

34) Butterworth RF, Norenberg MD, Felipo V, Ferenci P, Albrecht J, Blei AT, Members of the ICoEMoHE. Experimental models of hepatic encephalopathy: ISHEN guidelines. Liver Int 2009; 29:783-788.
35) Bernal W. Lactate is important in determining prognosis in acute liver failure. J Hepatol 2010;53:209-210.

36) Schurr A, Payne RS, Miller JJ, Rigor BM. Brain lactate is an obligatory aerobic energy substrate for functional recovery after hypoxia: further in vitro validation. J Neurochem 1997;69:423426.

37) Bosoi CR, Zwingmann $\mathrm{C}$, Marin $\mathrm{H}$, Parent-Robitaille $\mathrm{C}$, Huynh J, Tremblay M, et al. Increased brain lactate is central to the development of brain edema in rats with chronic liver disease. J Hepatol 2014;60:554-560.

38) Lundgaard I, Lu ML, Yang E, Peng W, Mestre H, Hitomi E, et al. Glymphatic clearance controls state-dependent changes in brain lactate concentration. J Cereb Blood Flow Metab 2016; doi:10.1177/0271678X16661202.

39) Duffy HS, Sorgen PL, Girvin ME, O’Donnell P, Coombs W, Taffet SM, et al. pH-dependent intramolecular binding and structure involving $\mathrm{Cx} 43$ cytoplasmic domains. J Biol Chem 2002;277:36706-36714.

40) Bosoi CR, Rose CF. Identifying the direct effects of ammonia on the brain. Metab Brain Dis 2009;24:95-102.

41) Bosoi CR, Parent-Robitaille C, Anderson K, Tremblay M, Rose CF. AST-120 (spherical carbon adsorbent) lowers ammonia levels and attenuates brain edema in bile duct-ligated rats. HePATOLOGY 2011;53:1995-2002.

42) Ye ZC, Oberheim N, Kettenmann H, Ransom BR. Pharmacological "cross-inhibition" of connexin hemichannels and swelling activated anion channels. Glia 2009;57:258-269.

43) Quist AP, Rhee SK, Lin H, Lal R. Physiological role of gapjunctional hemichannels. Extracellular calcium-dependent isosmotic volume regulation. J Cell Biol 2000;148:1063-1074.

44) Guevara M, Bru C, Gines P, Fernandez-Esparrach G, Sort P, Bataller R, et al. Increased cerebrovascular resistance in cirrhotic patients with ascites. Hepatology 1998;28:39-44.

45) Larsen FS, Olsen KS, Ejlersen E, Hansen BA, Paulson OB, Knudsen GM. Cerebral blood flow autoregulation and transcranial Doppler sonography in patients with cirrhosis. HePatology 1995;22:730-736.

46) Philips BJ, Armstrong IR, Pollock A, Lee A. Cerebral blood flow and metabolism in patients with chronic liver disease undergoing orthotopic liver transplantation. HePaTOLOGY 1998;27:369-376.

47) Sawhney R, Holland-Fischer P, Rosselli M, Mookerjee RP, Agarwal B, Jalan R. Role of ammonia, inflammation, and cerebral oxygenation in brain dysfunction of acute-on-chronic liver failure patients. Liver Transpl 2016;22:732-742.

Author names in bold designate shared co-first authorship.

\section{Supporting Information}

Additional Supporting Information may be found at onlinelibrary.wiley.com/doi/10.1002/hep.29031/suppinfo 